\title{
Aniridia-intellectual disability syndrome
}

INSERM

\section{Source}

INSERM. (1999). Orphanet: an online rare disease and orphan drug data base. Aniridiaintellectual disability syndrome. ORPHA:1068

Aniridia-intellectual disability syndrome is an extremely rare autosomal dominant developmental defect of the eye described in several members of one family that is characterized by the association of moderate intellectual disability with aniridia, lens dislocation, optic nerve hypoplasia (see this term) and cataracts. There have been no further descriptions in the literature since 1974. 